\title{
Prevalence of human papilloma virus genotypes 16, 18 in women with abnormal cervical cytology smears (abnormal Pap smear) attending Erbil Maternity Teaching Hospital
}

Accepted: 16/5/2019

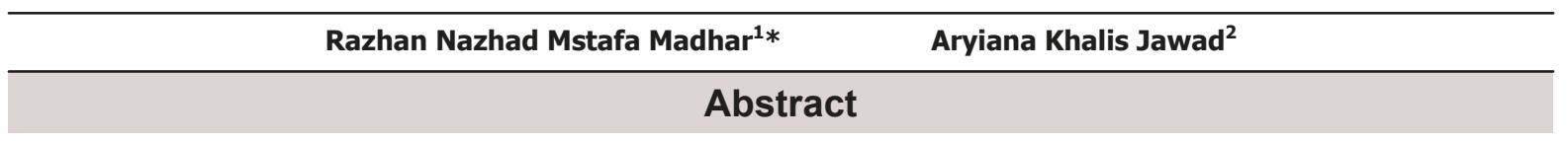

Background and objective: Cervical cancer is the fourth most common cancer among women. Carcinogenic Human Papilloma Virus (HPV) infection is the major etiological agent for cervical cancer, of which70\% are caused by HPV-16 and HPV-18 genotype infection. This study aimed to detect high risk $\operatorname{HPV}(16,18)$ in women with different abnormalities in pap smear to allow subsequent more accurate management of the cases and provide information that assists in future development of guidelines in our hospital.

Methods: We performed a cross-sectional study on 100 ladies with an abnormal pap smear. Subjects were interviewed and underwent cervical testing with broom using liquid based samples to confirm the cytological abnormality and test for HPV genotypes 16 and 18 using Polymerase Chain Reaction (PCR). Then, colposcopy referral was done according to local protocols.

Results: The median age of the subjects was 34. The prevalence of HPV genotypes 16,18 in the whole studied sample was $36 \%$. Various studied risk factors showed significant association with abnormal pap smear and positive HPV genotypes 16,18, including the number of partners, increasing parity, age at first pregnancy, and smoking. Data were analyzed by the statistical package for the social sciences (version 23).

Conclusion: The prevalence of HPV 16,18 was $36 \%$ in the sample, with the highest proportion being among those with high-grade lesions. Those using natural methods of contraception had higher rates of infection in comparison with other methods.

Keywords: Human Papillomavirus; Abnormal pap smear; Atypical squamous cell of undetermined significance; Low grade squamous intraepithelial lesion; High grade squamous intraepithelial lesion.

\section{Introduction}

Cervical cancer is the fourth most common cancer among women, after breast, colorectal, and lung cancer. ${ }^{1}$ About $80 \%$ of new cases of cervical cancer occur in developing countries. $^{2}$ Oncogenic HPV infection is the major etiological agent of cervical cancer, of which $70 \%$ are caused by HPV-16 and HPV-18 type. ${ }^{2,3}$ It may take up to 5-10 years for the infection to progress to the precancerous lesion. ${ }^{3}$ HPV genotype distribution varies with the severity of the cervical disease, patient demographics such as age, as well as geographical location. ${ }^{4,5}$ There has been a large decline in cervical cancer incidence and death rate in countries with regular cervical screening program during the past few decades. Unfortunately, there is no such decline in countries without such a screening program. The disproportionate burden of cervical cancer in developing countries with poor health systems is mainly due to a lack of effective screening program. ${ }^{2}$ In developing countries, limited access to effective screening means that the disease is often not identified until it is further advanced and symptoms develop. In addition, prospects for the treatment of such late-stage disease may be poor, resulting in a higher rate of death from cervical cancer in these countries. ${ }^{6}$

${ }^{1}$ Department of Obstetrics and Gynecology, College of Medicine, Hawler Medical University, Erbil, Iraq.

${ }^{2}$ Department of Obstetrics and Gynecology, Maternity Teaching Hospital, Erbil, Iraq.

* Correspondence: razhan_1989@yahoo.com 
The high mortality rate from cervical cancer globally $(52 \%)$ could be reduced by effective screening and treatment programs. ${ }^{6}$ Despite increased research into HPV, and the introduction of HPV vaccines to prevent infection and subsequent cervical cancer development, the screening will remain important. ${ }^{7-9}$ However, improved screening methods have also introduced some confusion, even controversy. ${ }^{8}$ HPV testing is more sensitive than the Pap test for detecting precancerous lesions. ${ }^{10}$ The HPV test identifies viruses that can cause cancer. On the other hand, some gynecologists still in use of Pap smear (plus HPV testing) as they believe that unknown cancer causing viruses can be missed by HPV test only. However, reports of rare HPV-negative, Pap-test-positive cancers are motivating ongoing use of both tests (co-testing) despite the high costs.5 The accumulated evidence supports the inclusion of HPV testing in screening; thus, the main choice moving forward is between cotesting and primary HPV testing only. ${ }^{10}$ To the best knowledge of the researchers of this article, this is the first time to test for HPV in a sample of Kurdish women. Therefore, this study aimed to detect high risk HPV types $(16,18)$ in women presenting with different abnormalities in the pap smear to allow subsequent more accurate management of the cases. The study provides information that may help in the future development of guidelines in our community in light of the lack of regular cervical screening programs in our community.

\section{Methods}

\section{Design and setting of the study}

This cross-sectional study was carried out in a group of women attending the Maternity Teaching Hospital in Erbil city, Kurdistan region of Iraq, from June 2017 to September 2018.

\section{Sampling method and sample size:}

A sample size of 94 women was calculated based on having a $10 \%$ accepted margin of error around an estimated prevalence of HPV type 16 of $41.9 \%$ in women with cervical cytological abnormality with a $95 \%$ confidence interval, ${ }^{12}$ and the sample size had been increased to 100 . The women who underwent Pap smear examination in Maternity teaching hospital and had cervical cytological abnormality were selected to participate in the study until the required sample size gained. The inclusion criteria included all married women between 21-65 years of age, including those who had a history of chronic medical illnesses like diabetes and hypertension. This age group was included according to the guidelines of cervical screening, which includes this age group. The exclusion criteria included women who had hysterectomy for causes other than cervical cytological abnormalities, women younger than 21, and women older than 65. Unmarried women were also not included in the study for social and cultural reasons and those who refused to give consent.

\section{Data collection}

The sample collection was carried out from September 2017 to September 2018. It was preceded at the time of sample collection by an interview using a structured epidemiological questionnaire that included information on sociodemographic characteristics, habits, and sexual and reproductive history. The patients who have been classified as being in the inclusion group were educated about the precautions that are recommended before taking the cervical smear (liquid based sample collection), including not being in menstrual period and avoiding sexual intercourse, douching, and using spermicidal products the day before the test. The patient was asked to lie supine, in a dorsal lithotomy position to correctly perform a pap smear. The coccyx of the patient was at the edge of the examination table to provide adequate visualization of the cervix once the speculum is inserted. After adequate positioning, careful clinical examination of 
the external genitalia, and the perianal region was done. Then, the speculum was inserted, and an adequate sample was obtained from the cervix using a broom like device. The central bristles of the broom was inserted into the endocervix deep enough to allow the shorter bristles to fully contact the ectocervix, then pushing and rotating the broom in a clockwise direction five times. The broom was then rinsed as quickly as possible into alcohol based liquid container (the thinprep pap test container) by pushing the broom into the bottom of the container 10 times. The cap of the container was tightened, and the patient's name and ID number were recorded on the container then patient information and medical history were recorded on the cytology requisition form. The sample container and the requisition form were transported to the laboratory.

\section{Cytological examination and HPV analysis}

Cytological smears consisted of collected cells from the transformational zone (squamo-columnar junction) using the broom. After appropriate collection of the samples, results for both the pap smear and HPV detection were reported using the same samples at the cytological and histopathological department of the laboratory in Maternity teaching hospital. The results of the samples and the degree of cervical abnormalities were interpreted according to the Bethesda System revised in 2001. The extraction of the genomic DNA from the samples was performed using PCR. The PCR results were reported as positive for HPV16, positive for HPV18, positive for HPV 16,18 or negative for HPV 16,18 .

\section{Ethical approval}

Ethical approval to conduct this study was obtained from the Scientific Council of Obstetrics and Gynecology/Kurdistan Board for Medical Specialties. All interviews performed were in accordance with the ethical standards of the institutional research committee. Informed consent was obtained from each woman who agreed to participate in the study. Participants were assured that confidentiality would be maintained and that their information would only be used for research purposes.

\section{Statistical analysis}

Data were analyzed by the statistical package for the social sciences (SPSS, version 23). Means and proportions were displayed. The student's t-test was used to compare means, and the Chi-square test or Fisher's Exact test was used for the comparison of categorical variables as appropriate. A $P$ value of $\leq 0.05$ was considered significant.

\section{Results}

Hundred ladies with abnormal Pap smear results had been included in the study. Their mean age $\pm S D$ was $36.5 \pm 8.5$ years, with a median of 34 years. The age range was 24 to 53 years. The results showed that $7 \%$ of the sample aged $35-39$ years, $12 \%$ aged $40-44$ years, while the rest of the age group represents $27 \%$. The outcome shows that the majority $(84 \%)$ of the women started their sexual activity at 17 years or over, and $(88 \%)$ had only one sexual partner throughout their life. The majority $(84 \%)$ of the studied sample used some sorts of contraception throughout their life, and $19 \%$ were smokers (Table 1), $36 \%$ of the women had five children or more, and only $8 \%$ became pregnant before the age of 17 years. 
Prevalence of Human Papilloma Virus Genotypes ......

Table 1: Basic characteristics of the studied sample.

\begin{tabular}{|c|c|c|}
\hline Characteristic & No. & $(\%)$ \\
\hline \multicolumn{3}{|l|}{ Age (years) } \\
\hline$<30$ & 27 & $(27.00)$ \\
\hline $30-34$ & 27 & $(27.00)$ \\
\hline $35-39$ & 7 & 7.00 \\
\hline $40-44$ & 12 & 12.00 \\
\hline$\geq 45$ & 27 & 27.00 \\
\hline \multicolumn{3}{|c|}{ Initiation of sexual activity (years) } \\
\hline$<17$ & 16 & 16.00 \\
\hline$\geq 17$ & 84 & 84.00 \\
\hline \multicolumn{3}{|c|}{ Number of sexual partners in lifetime } \\
\hline One & 88 & 88.00 \\
\hline Two & 12 & 12.00 \\
\hline \multicolumn{3}{|c|}{ Number of children born } \\
\hline 0 & 4 & 4.00 \\
\hline $1-4$ & 60 & 60.00 \\
\hline$\geq 5$ & 36 & 36.00 \\
\hline \multicolumn{3}{|c|}{ Age of first pregnancy (years) } \\
\hline$<17$ & 8 & 8.00 \\
\hline$\geq 17$ & 92 & 92.00 \\
\hline \multicolumn{3}{|c|}{ Ever use of contraception } \\
\hline Yes & 84 & 84.00 \\
\hline No & 16 & 16.00 \\
\hline \multicolumn{3}{|l|}{ Smoking } \\
\hline Yes & 19 & 19.00 \\
\hline No & 81 & 81.00 \\
\hline Total & 100 & 100.00 \\
\hline
\end{tabular}


Table 2 shows that the prevalence of HPV in the whole sample was $36 \%$. No significant association was detected between the prevalence of HPV and the Pap smear results $(P=0.140)$. However, it is worth mentioning that the highest rates were among those with high grade squamous intraepithelial lesion (HSIL) (50\%) and those with the atypical squamous cell of undetermined significance (ASCUS) (44\%). The prevalence of HPV was highest among women using the natural methods of contraception $(62.5 \%)$, while none of those using condoms tested positive for HPV $(P<0.001)$. Half of those with a history of gonococcal infection tested positive for HPV, and none of those with a trichomonas infection history tested positive. The highest prevalence (62.5\%) was among those with unknown history for STIs $(P<0.001)$. The prevalence of HPV was $100 \%$ among those with a family history of breast cancer, compared with $36.8 \%$ among those with no cancer history $(P<0.001)$.

Table 2: Prevalence of HPV by some risk factors.

\begin{tabular}{|c|c|c|c|c|c|c|c|}
\hline & \multicolumn{4}{|c|}{ Prevalence of HPV } & \multirow{2}{*}{\multicolumn{2}{|c|}{ Total }} & \multirow[b]{3}{*}{$P$ value } \\
\hline & \multicolumn{2}{|c|}{ Positive } & \multicolumn{2}{|c|}{ Negative } & & & \\
\hline & No. & (\%) & No. & $(\%)$ & \multicolumn{2}{|c|}{ No. $\quad(\%)$} & \\
\hline \multicolumn{8}{|l|}{ Pap smear } \\
\hline ASCUS & 16 & $(44.40)$ & 20 & $(55.60)$ & 36 & $(100.00)$ & \\
\hline LSIL & 8 & (22.20) & 28 & $(77.80)$ & 36 & $(100.00)$ & \\
\hline HSIL & 8 & $(50.00)$ & 8 & $(50.00)$ & 16 & $(100.00)$ & \\
\hline Severe cervicitis & 4 & $(33.30)$ & 8 & $(66.70)$ & 12 & $(100.00)$ & 0.140 \\
\hline \multicolumn{8}{|l|}{ Contraception } \\
\hline OCP & 8 & $(28.60)$ & 20 & $(71.40)$ & 28 & $(100.00)$ & \\
\hline Condom & 0 & $(0.00)$ & 16 & $(100.00)$ & 16 & $(100.00)$ & \\
\hline Natural & 20 & $(62.50)$ & 12 & $(37.50)$ & 32 & $(100.00)$ & \\
\hline Implants & 4 & $(50.00)$ & 4 & $(50.00)$ & 8 & $(100.00)$ & \\
\hline None & 4 & $(25.00)$ & 12 & $(75.00)$ & 16 & $(100.00)$ & $<0.001$ \\
\hline \multicolumn{8}{|l|}{ History of STIs } \\
\hline Gonococcal infection & 4 & $(50.00)$ & 4 & $(50.00)$ & 8 & $(100.00)$ & \\
\hline Trichomonus & 0 & $(0.00)$ & 4 & $(100.00)$ & 4 & $(100.00)$ & \\
\hline None & 12 & $(21.40)$ & 44 & $(78.60)$ & 56 & $(100.00)$ & \\
\hline Unknown & 20 & $(62.50)$ & 12 & $(37.50)$ & 32 & $(100.00)$ & $<0.001^{*}$ \\
\hline \multicolumn{8}{|l|}{ Family history of CA } \\
\hline Cervical CA & 0 & $(0.00)$ & 4 & $(100.00)$ & 4 & $(100.00)$ & \\
\hline Endometrial CA & 0 & $(0.00)$ & 8 & $(100.00)$ & 8 & $(100.00)$ & \\
\hline Breast CA & 8 & $(100.00)$ & 0 & $(0.00)$ & 8 & $(100.00)$ & \\
\hline Others & 0 & $(0.00)$ & 4 & $(100.00)$ & 4 & $(100.00)$ & \\
\hline None & 28 & $(36.80)$ & 48 & $(63.20)$ & 76 & $(100.00)$ & $<0.001^{*}$ \\
\hline Total & 36 & $(36.00)$ & 64 & $(64.00)$ & 100 & $(100.00)$ & \\
\hline
\end{tabular}

*By Fisher's exact test.

Abbreviations: ASCUS = Atypical squamous cell of undetermined significance. LSIL = Low grade intraepithelial lesion. HSIL = High grade intraepithelial lesion. STIs = Sexually transmitted infections. 
Table 3 shows no significant association between age and prevalence of HPV $(P=0.745)$. The table shows that $100 \%$ of women whose first pregnancy was before 17 tested positive for HPV $(P<0.001)$. The prevalence of HPV was significantly $(P=0.026)$ higher among those with two partners $(66.7 \%)$ than among those with one partner $(31.8 \%)$. No significant association was detected between the prevalence and the age of marriage $(P=0.317)$. The highest prevalence $(100 \%)$ was among the nulliparous women $(P=0.036)$, but it is evident that only four women were nulliparous. The prevalence among smokers $(63.2 \%)$ was significantly higher than the prevalence $(29.6 \%)$ among the non-smokers $(P=0.006)$.

Table 3: Prevalence of HPV by age and some social behaviors.

\begin{tabular}{|c|c|c|c|c|c|c|c|}
\hline & \multicolumn{2}{|c|}{ Positive } & \multicolumn{2}{|c|}{ negative } & \multicolumn{2}{|c|}{ Total } & \multirow[t]{2}{*}{$P$ value } \\
\hline & No. & $(\%)$ & No. & $(\%)$ & No. & $(\%)$ & \\
\hline \multicolumn{8}{|c|}{ Age (years) } \\
\hline$<30$ & 11 & $(40.70)$ & 16 & $(59.30)$ & 27 & $(100.00)$ & \multirow{5}{*}{$0.745^{*}$} \\
\hline $30-34$ & 7 & $(25.90)$ & 20 & (74.10) & 27 & $(100.00)$ & \\
\hline $35-39$ & 3 & $(42.90)$ & 4 & $(57.10)$ & 7 & $(100.00)$ & \\
\hline $40-44$ & 4 & $(33.30)$ & 8 & $(66.70)$ & 12 & $(100.00)$ & \\
\hline$\geq 45$ & 11 & $(40.70)$ & 16 & $(59.30)$ & 27 & $(100.00)$ & \\
\hline \multicolumn{8}{|c|}{ Age at first pregnancy } \\
\hline$<17$ & 8 & $(100.00)$ & 0 & $(0.00)$ & 8 & $(100.00)$ & \multirow{2}{*}{$<0.001^{*}$} \\
\hline$\geq 17$ & 28 & $(30.40)$ & 64 & $(69.60)$ & 92 & $(100.00)$ & \\
\hline \multicolumn{8}{|c|}{ Number of partners } \\
\hline One & 28 & $(31.80)$ & 60 & $(68.20)$ & 88 & $(100.00)$ & \multirow{2}{*}{$0.026^{*}$} \\
\hline Two & 8 & $(66.70)$ & 4 & $(33.30)$ & 12 & $(100.00)$ & \\
\hline \multicolumn{8}{|c|}{ Age of marriage } \\
\hline$<17$ & 4 & $(25.00)$ & 12 & $(75.00)$ & 16 & $(100.00)$ & \multirow{2}{*}{0.317} \\
\hline$\geq 17$ & 32 & $(38.10)$ & 52 & $(61.90)$ & 84 & $(100.00)$ & \\
\hline \multicolumn{8}{|l|}{ Parity } \\
\hline 0 & 4 & $(100.00)$ & 0 & $(0.00)$ & 4 & (100.00) & \multirow{3}{*}{$0.036^{*}$} \\
\hline $1-4$ & 20 & (33.30) & 40 & $(66.70)$ & 60 & (100.00) & \\
\hline$\geq 5$ & 12 & $(33.30)$ & 24 & $(66.70)$ & 36 & $(100.00)$ & \\
\hline \multicolumn{8}{|c|}{ Smoking } \\
\hline No & 24 & $(29.60)$ & 57 & $(70.40)$ & 81 & $(100.00)$ & \multirow{3}{*}{0.006} \\
\hline Yes & 12 & $(63.20)$ & 7 & $(36.80)$ & 19 & $(100.00)$ & \\
\hline Total & 36 & $(36.00)$ & 64 & $(64.00)$ & 100 & $(100.00)$ & \\
\hline
\end{tabular}

*By Fisher's exact test. 
It is evident in Table 4 that all the patients aged $\geq 45$ years had HPV 16, and all of the patients aged 35-44 years were positive for HPV 16 and $18 P<0.001)$. All those with severe cervicitis were positive for HPV 16.
There was a significant association between Pap smear results and type of $\operatorname{HPV}(P=0.010)$. No significant association was detected between the HSIL diagnosis with the type of HPV $(P=0.227)$.

Table 4: Prevalence of HPV subtypes by age, Pap smear results and HSIL.

\begin{tabular}{|c|c|c|c|c|c|c|c|c|c|}
\hline & \multicolumn{2}{|c|}{ HPV16 } & \multicolumn{2}{|c|}{ HPV18 } & \multicolumn{2}{|c|}{ HPV $16 \& 18$} & \multicolumn{2}{|c|}{ Total } & \multirow[t]{2}{*}{$P$ value } \\
\hline & No. & (\%) & No. & (\%) & No. & (\%) & No. & (\%) & \\
\hline \multicolumn{10}{|l|}{ Age } \\
\hline$<30$ & 1 & $(9.10)$ & 8 & (72.70) & 2 & (18.20 & 11 & (100.00) & \\
\hline $30-34$ & 4 & $(57.10)$ & 0 & $(0.00)$ & 3 & $(42.90$ & 7 & (100.00) & \\
\hline $35-39$ & 0 & $(0.00)$ & 0 & $(0.00)$ & 3 & $(100.00)$ & 3 & (100.00) & $<0.001^{*}$ \\
\hline $40-44$ & 0 & $(0.00)$ & 0 & $(0.00)$ & 4 & (100.00) & 4 & (100.00) & \\
\hline$\geq 45$ & 11 & $(100.00)$ & 0 & $(0.00)$ & 0 & $(0.00)$ & 11 & (100.00) & \\
\hline \multicolumn{10}{|c|}{ Pap smear } \\
\hline ASCUS & 4 & $(25.00)$ & 4 & $(25.00)$ & 8 & $(50.00)$ & 16 & (100.00) & \multirow{4}{*}{$0.010^{*}$} \\
\hline LSIL & 4 & $(50.00)$ & 4 & $(50.00)$ & 0 & $(0.00)$ & 8 & (100.00) & \\
\hline HSIL & 4 & $(50.00)$ & 0 & $(0.00)$ & 4 & $(50.00)$ & 8 & (100.00) & \\
\hline $\begin{array}{l}\text { Severe } \\
\text { cervicitis }\end{array}$ & 4 & $(100.00)$ & 0 & $(0.00)$ & 0 & $(0.00)$ & 4 & (100.00) & \\
\hline \multicolumn{10}{|l|}{ HSIL } \\
\hline No & 12 & $(42.90)$ & 8 & $(28.60)$ & 8 & $(28.60)$ & 28 & (100.00) & \\
\hline Yes & 4 & $(50.00)$ & 0 & $(0.00)$ & 4 & $(50.00)$ & 8 & $(100.00)$ & $0.227^{*}$ \\
\hline Total & 16 & $(44.40)$ & 8 & (22.20) & 12 & (33.30) & 36 & (100.00) & \\
\hline
\end{tabular}

${ }^{*} P$ calculated by Fisher's exact test. 


\section{Discussion}

Our prospective study concluded that the prevalence of HPV 16,18 was $36 \%$ in the whole population, and the highest proportion was among those with high grade lesions (HSIL). Those using natural contraception methods also had higher rates of infection compared with other methods, and cases with a history of gonococcal infection were positive for HPV 16 or 18 in half of them. A higher prevalence has been found in a study that had been carried out in Australia, Melbourne in 2009 which showed that overall $83.9 \%$ of women with an abnormal pap smear, had one of the high risk HPV infections. ${ }^{5}$ On the other hand, another study which was done in Turkey showed that overall prevalence of HPV in women with abnormal pap smear was $36 \%$ and HPV 16 infection being the commonest. ${ }^{13}$ The prevalence of HPV was highest among those younger than 35 years old, although it was statistically not significant. A study done in the UK showed that women below 30 years were more likely to have HPV infection. ${ }^{14}$ Such difference in the two results may be due to earlier diagnosis and intervention in the second population group (UK). Despite the nonsignificant association, HSIL was associated with the highest positivity rate for either subtype 16 or $18 \mathrm{HPV}$ infection. The association may be limited by the sample size, and bigger sample size may solve this problem. These findings were in conformity with the data given by the University of Pittsburg (Infectious Diseases and Microbiology Graduate School of Public Health). According to them, high risk HPV was positive in $79-90 \%$ cases of $\mathrm{HSIL}^{13}$ Condom use appears to offer relatively good protection from HPV infections and associated cervical neoplasia. ${ }^{16}$ This was obvious in our study as none of those using condoms had been tested positive for HPV 16 or 18 . The current study findings were comparable with a systemic review performed on condom use in the prevention of HPV infection and cervical neoplasia by Department of Public Health, University of Copenhagen. ${ }^{15}$ Although HPV infection is the commonest type of sexually transmitted infections (STI) in many parts of the world, there is an association of HPV infection with other STIs. ${ }^{16}$ In our study, $50 \%$ of those with gonococcal infection tested positive for either HPV 16 or 18. However, the lack of specialized STI clinics in our area makes reporting the prevalence of STIs and their association with HPV infection very difficult. According to a longitudinal cohort study of American female college students, initiation of sexual intercourse at an early age makes the possibility of being infected with HPV more likely than later sexual initiation in part because of certain behavioral factors and partner characteristics. ${ }^{17}$ This study found a significant association between the age of first pregnancy and HPV infection in the studied population. Those with the age of first pregnancy less than 17 have $100 \%$ rate of being positive for either HPV 16 or 18. In light of these findings, it is suggested that providers encourage adolescents who have not yet had sexual intercourse to postpone sexual initiation. It is also recommended that youth who had their first sexual experience at early age should benefit from counseling that stresses on the importance of safe sex practices including barriers such as condoms and dental dams. ${ }^{17}$ To our knowledge, this is the first cross-sectional study on the prevalence of HPV $(16,18)$ in women with abnormal pap smear in Erbil city. The study was conducted in Erbil Maternity Teaching Hospital, the only governmental hospital in Erbil city serving obstetric and gynecologic cases. Even patients who have been seen in private clinics would be referred to the cervical pathology unit of this hospital for further assessments. This makes the population that has been studied a good representative sample of those with an abnormal pap smear. These points make the study unique in this area of research at the moment. As a limitation in this study, we did not correlate the 
cytological changes and HPV results with the colposcopy appearance of the cervix and histological changes. Such limitation is because HPV triage has become a new way for follow up of patients with some confusing borderline changes in the pap smear, so colposcopy has not necessarily been done for all patients. Future studies should address this issue to provide further information and classification of the cervical pathology to help reform local protocols in our hospital. HPV vaccine for the prevention of some HPV genotypes has been approved by US Food and Drug Administration for women between 9-26 years of age in $2006 .{ }^{18}$ In the background of our research result, vaccination is recommended for women in our community in the hope of preventing HPV infection.

\section{Conclusion}

The prevalence of HPV 16,18 was $36 \%$ in the sample, with the highest proportion being among those with high grade lesions (HGSIL). Those using natural methods of contraception had higher rates of infection in comparison with other methods.

\section{Competing interests}

The authors declare no competing interests.

\section{References}

1. Bray F, Ferlay J, Soerjomataraml, Siegel RL, Torre LA, Jemal A. Global Cancer Statistics 2018: GLOBOCAN Estimates of Incidence and Mortality Worldwide for 36 Cancers in 185 Countries. Ca Cancer J Clin 2018; 68:394-424.

2. Muñoz N, Bosch FX, Castellsagué $X$, Díaz $M$, de Sanjose S, Hammouda D, et al, Against which human papillomavirus types shall we vaccinate and screen? The international perspective. Int J Cancer 2004; 111(2):278-85.

3. Schiffman M, Castle PE, Jeronimo J, Rodriguez AC, Wacholder S.Human papillomavirus and cervical cancer.Lancet 2007; 370(9590):890-907.

4. Munoz N, Bosch FX, de Sanjose S, Herrero R, Castellsagué $\mathrm{X}$, Shah KV. International Agency for Research on Cancer Multicenter Cervical Cancer Study Group. Epidemiologic classification of human papillomavirus types associated with cervical cancer. N Engl J Med 2003; 348:518-27.

5. Stevens MP, Garland SM, Tan JH, Quinn MA, Petersen RW, Tabrizi SN. HPV genotype prevalence in women with abnormal pap smears in Melbourne, Australia. J Med Virol 2009; 81(7):1283-91.

6. World Health Organization. Comprehensive cervical cancer control. A guide to essential practice. Geneva. (Accessed September 19, 2018, at http://www.who.int/news-room/factsheets/detail/human-papillomavirus-(hpv)-andcervical-cancer).

7. McGraw SL, Ferrante JM .Update on prevention and screening of cervical cancer. World J Clin Oncol 2014; 5(4):744-52.

8. Oxford University Press USA. HPV testing is better than the Pap test at detecting cervical cancer. (Accessed October 17, 2018, at https://www.eurekalert.org/pub_releases/201711/oupu-hti111317.php).

9. Franco EL, Cuzick J, Hildesheim A,de Sanjosé S. Issues in planning cervical cancer screening in the era of HPV vaccination. Vaccine 2006; 24(Suppl 3):S171-7.

10. Schiffman M, Kinney WK, Cheung LC, Gage JC, Fetterman B, Poitras NE. Relative Performance of HPV and Cytology Components of Cotesting in Cervical Screening. J Natl Cancer Inst 2018; 110(5):501-8.

11. Ogilvie GS, van Niekerk $D$, Krajden $M$, Smith LW, Cook D, Gondara L, et al. Effect of Screening With Primary Cervical HPV Testing vs Cytology Testing on High-grade Cervical Intraepithelial Neoplasia at 48 Months: The HPV FOCAL Randomized Clinical Trial. JAMA 2018; 320(1):43-52.

12. Youssef MA, Abdelsalam L, Harfoush RA, Talaat IM, Elkattan E, Mohey A,et al. prevalence of human papilloma virus (HPV) and its genotypes in cervical specimens of Egyptian women by linear array HPV genotyping test.Infect Agent Cancer 2016; 11:6.

13. Dursun $P$, Senger $S$, Arsalan $H$, Kuscu E, Ayhan A. Human papillomavirus (HPV) prevalence and types among Turkish women at a gynecology outpatient unit. BMC Infectious Diseases 2009; 9:191.

14. Sargent A, Bailey A, Almonte M, Turner A, Thomson V, Pito J, et al. Prevalence of type specific HPV infection by age and grade of cervical cytology:data from ARTISTIC trial. British journal of cancer. $\mathrm{Br} \mathrm{J}$ Cancer 2008; 98(10):1704-9.

15. Lam JU, Rebolj M, Dugué PA, Bonde J, von Euler-Chelpin M, Lynge E.Condom use in prevention of Human Papillomavirus infections and cervical neoplasia: systematic review of longitudinal studies. J Med Screen 2014; 21(1):38-50.

16. Kim HS, Kim TJ, Lee IH, Hong SR. Associations between sexually transmitted infections, high-risk human papillomavirus infection, and abnormal cervical Pap smear results in OB/GYN outpatients. J Gynecol Oncol 2016; 27(5):e49. 
Prevalence of Human Papilloma Virus Genotypes ......

Zanco J. Med. Sci., Vol. 24, No. (2), August, 2020

https://doi.org/10.15218/zjms.2020.025

17. Kahn JA, Rosenthal SL, Succop PA, Ho GY, Burk RD. Mediators of the association between age of first sexual intercourse and subsequent human papillomavirus infection. Pediatrics 2002; 109(1):E5.

18. Roden R, Wu TC. How will HPV vaccines affect cervical cancer? Nat Rev Cancer 2006; 6(10):753 $-63$. 